\section{ORIGINAL RESEARCH}

M. Neema

Z.D. Guss

J.M. Stankiewicz

A. Arora

B.C. Healy

R. Bakshi

\title{
Normal Findings on Brain Fluid-Attenuated Inversion Recovery MR Images at 3T
}

BACKGROUND AND PURPOSE: Fluid attenuated inversion recovery (FLAIR) MR imaging of the brain has become a routine tool for assessing lesions in patients with suspected neurologic disorders. There is growing interest in 3 T brain FLAIR MR imaging but little normative data are available. The purpose of this study was to evaluate the frequency and topography of cerebral hyperintensities seen with FLAIR MR imaging of the brain at $3 \mathrm{~T}$ in a normal population and compare those findings to $1.5 \mathrm{~T}$.

MATERIALS AND METHODS: Whole-brain 2D FLAIR MR imaging was performed in 22 healthy controls (mean age, $44 \pm 8$ years; range, 30-53 years) at 3T. Fifteen of these subjects also underwent 2D FLAIR at 1.5T, with similar optimized parameters and voxel size. Cerebral hyperintense areas, including discrete foci, anterior and posterior periventricular capping, diffuse parenchymal hyperintensity, septal hyperintensity, corticospinal tract hyperintensity, and CSF flow artifacts were assessed. The Spearman rank test assessed the correlation between discrete hyperintense foci and age. The Wilcoxon signed rank test compared foci detectability at $3 \mathrm{~T}$ versus $1.5 \mathrm{~T}$.

RESULTS: FLAIR at 3T commonly showed hyperintensities such as discrete foci (mean, 10.68 per subject; at least 1 present in $68 \%$ of subjects), anterior and posterior periventricular capping, diffuse posterior white matter hyperintensity, septal hyperintensity, corticospinal tract hyperintensity, and ventricular CSF flow artifacts. FLAIR at 3T showed a higher hyperintense foci volume $(170 \pm 243$ versus $\left.93 \pm 152 \mathrm{~mm}^{3}, P<.01\right)$ and number $(9.4 \pm 13$ versus $5.5 \pm 9.2, P<.01)$ than at $1.5 \mathrm{~T}$. No significant differences $(P=.68)$ in the length/diameter of individual discrete hyperintense foci were seen between 3T and 1.5T. Discrete foci volume ( $r=0.72$ at 3T, $r=0.70$ at 1.5T) and number $(r=0.74$ at $3 \mathrm{~T} ; r=0.69$ at $1.5 \mathrm{~T}$ ) correlated with age to a similar degree on both platforms. All discrete foci were confined to the noncallosal supratentorial white matter. The other nonfocal hyperintensities (anterior and posterior periventricular capping, diffuse parenchymal hyperintensity, septal hyperintensity, corticospinal tract hyperintensity, and CSF flow artifacts) were generally more common and prominent at $3 \mathrm{~T}$ than at $1.5 \mathrm{~T}$.

CONCLUSIONS: Discrete and diffuse parenchymal brain white matter FLAIR hyperintensities are more common and prominent at $3 \mathrm{~T}$ than at $1.5 \mathrm{~T}$ in healthy volunteers.

$F^{\prime}$ luid-attenuated inversion recovery (FLAIR) MR imaging of the brain has become a routine tool for assessing lesions in patients with suspected neurologic disorders such as multiple sclerosis (MS). ${ }^{1,2}$ FLAIR is particularly useful for assessing supratentorial pathology but is less useful for assessing the posterior fossa and spinal cord. ${ }^{3}$ FLAIR hyperintensities seen in healthy subjects have been characterized at $1.5 \mathrm{~T},{ }^{4}$ but not at higher field strengths.

As interest in FLAIR has grown during the past decade, so too has interest in brain MR imaging at field strengths higher than 1.5T (eg, 3T and higher). The availability of high-field-

\section{Received November 5, 2008; accepted after revision December 11}

From the Departments of Neurology (M.N., Z.D.G., J.M.S., A.A., B.C.H., R.B.) and Radiology (R.B.), Brigham and Women's Hospital, Laboratory for Neuroimaging Research, Partners Multiple Sclerosis Center, Harvard Medical School, Boston, Mass.

This work was supported by research grants from the National Institutes of Health (NIH R01 NS055083-01) and National Multiple Sclerosis Society (RG3705A1, RG3798A2).

Previously presented at: 60th Annual Meeting of the American Academy of Neurology, April 12-19, 2009; Chicago, III; and at the first Joint Meeting of the Americas Committee on Treatment and Research in Multiple Sclerosis (ACTRIMS) and its counterparts in Europe and Latin America, ECTRIMS and LACTRIMS, September 17-20, 2008; Montréal, Ontario, Canada.

Please address correspondence to Rohit Bakshi, MD, Associate Professor of Neurology and Radiology, Brigham and Women's Hospital, Harvard Medical School, Laboratory for Neuroimaging Research, One Brookline PI, Ste 602, Brookline, MA 02445; e-mail: rbakshi@ bwh.harvard.edu

Indicates open access to non-subscribers at www.ajnr.org

DOI 10.3174/ajnr.A1514 strength scanners (ie, 3T and higher) has the potential to revolutionize research and clinical care in various neurologic disorders. ${ }^{2,5,6}$ Such higher field approaches have held the promise of increasing diagnostic sensitivity and specificity in a variety of neurologic conditions. For example, prior studies have detected a greater number of lesions at higher field strengths (3T and higher) than at $1.5 \mathrm{~T}$ in patients with MS. ${ }^{78}$ Another study demonstrated that not only does 3T MR imaging detect increased lesion volume compared with $1.5 \mathrm{~T} \mathrm{MR}$ imaging, but it can increase diagnostic yield in confirming MS. ${ }^{9}$ Furthermore, FLAIR diffusion tensor imaging uncovers anisotropic parenchymal tissue normally obscured by CSF signal intensity. ${ }^{10}$ With such profound opportunities in the transition to higher fields, it is critical to establish normative data to maximize diagnostic accuracy and specificity.

The present study had 3 principal aims: We first sought to describe both qualitatively and quantitatively brain hyperintensities detected in healthy volunteers with FLAIR at 3T. Second, we tested the hypothesis that FLAIR would show increased sensitivity to brain hyperintensities at 3T versus $1.5 \mathrm{~T}$. Finally, we tested the association between discrete hyperintense foci and age.

\section{Materials and Methods}

\section{Subject Characteristics and Image Acquisition}

We examined 22 adult healthy volunteers (mean age, $44 \pm 8$ years; range, $30-53$ years). This Health Insurance Portability and Accountability 
Act-compliant study included institutional review board (IRB) approval and informed consent. Participants for this study were recruited by using an IRB-approved advertisement that was posted in a local newspaper and our hospital Website. A telephone interview was conducted by using a questionnaire, and any potential participants with a history of major medical, neurologic, or neuropsychiatric disorders and current or previous history of substance abuse were excluded. For example, questions were asked to exclude subjects who had a history of hypertension, diabetes, head trauma, migraine, learning disabilities, depression, bipolar disorder, alcohol/tobacco/recreational drug abuse, or any conditions that would preclude MR imaging. Regarding tobacco, we excluded any individuals with current or recent (within 5 years) use. Entry into the study was on the basis of patient-reported history: no formal bedside physical or neurologic examination or neuropsychiatric testing was performed. Imaging of the brain was performed in 15 of these subjects (mean age, $43 \pm 8$ years; range, $30-53$ years) at both $1.5 \mathrm{~T}$ and $3 \mathrm{~T}$; an additional 7 subjects were interested in having only 1 scan due to the time commitment and thus were scanned at 3T only. There was no significant $(P=$ .75 ) age difference between the subject groups studied at 1.5T and 3T. Subjects underwent 2D fast FLAIR MR imaging of the brain; technical details on the scan protocol are provided in Table 1. Due to the potential at $3 \mathrm{~T}$ to exceed specific absorption rate, patient safety limitations, and scanning time considerations, TR, TE, and echo-train length varied between the 2 platforms, though voxel size was nearly equivalent. ${ }^{11}$

Due to a change in scanning protocol early in the project, the section thickness for the scans was slightly variable between but generally not within subjects. Of the total 22 subjects scanned at 3T, 18 were scanned with the main protocol shown in Table 1 by using 2-mm-thick axial sections and 4 were scanned with a similar protocol by using a $3-\mathrm{mm}$ section thickness. Of the 15 subjects scanned at $1.5 \mathrm{~T}$, 14 were scanned with the $2-\mathrm{mm}$ protocol and 1 subject was scanned with the 3-mm protocol. Only 1 subject scanned at both $1.5 \mathrm{~T}$ and $3 \mathrm{~T}$ had variable section thicknesses between platforms; but because this subject did not show any discrete hyperintense foci, we did not exclude this subject from the analysis. Otherwise, the scanning protocol for the 22 subjects was similar within and across subjects.

\begin{tabular}{lcc}
\hline \multicolumn{3}{l}{ Table 1: MR imaging protocol and technique } \\
\hline Parameters & $1.5 T$ & $3 T$ \\
\hline Head coil & Quadrature & Receive-only phased array \\
Number of channels & 4 & 8 \\
Pulse sequence & $2 D$ fast FLAIR & 2 D fast FLAIR \\
FOV (cm) & $24 \times 24$ & $25 \times 25$ \\
Matrix size & $256 \times 256$ & $256 \times 256$ \\
Section thickness & 2 (no gap) & 2 (no gap) \\
$\quad(m m)$ & 10,000 & \\
TR (ms) & 125 & 9,000 \\
TE (ms) & 2700 & 157 \\
Inversion time (ms) & 3 & 2250 \\
NSA & $90^{\circ}$ & 1 \\
Flip angle & 8.2 & $90^{\circ}$ \\
Pixel size (mm) & $0.938 \times 038 \times 2$ & $0.976 \times 0.976 \times 2$ \\
Scanning time (min) & 0.9 .1 \\
\hline
\end{tabular}

Note:-NSA indicates number of signal averages; FLAIR, fluid-attenuated inversion recovery.

\section{Image Analysis}

Image analysis was performed by using the software package Jim, Version 3.0 (Xinapse Systems, Northants, UK; http://www.xinapse. com). Scans were anonymized and randomized for analysis. Discrete areas of increased signal-intensity abnormality on FLAIR images, referred to as "hyperintense foci," were identified by the consensus of 3 trained observers and confirmed by an experienced observer to resolve any discrepancies. We avoided using the term "lesions" to describe the foci because of the normative population being studied. The number and volume of FLAIR hyperintense discrete foci were assessed through an edge-finding tool based on local thresholding that was applied to each axial section to identify discrete foci contours. Manual adjustments were applied where necessary. The maximum width or length of each hyperintense focus and the anterior hyperintense capping of the ventricles were assessed with a measurement tool. Hyperintense areas not well suited to quantitative analysis, such as diffuse white matter hyperintensity, septal hyperintensity, ${ }^{4}$ corticospinal tract hyperintensity, ${ }^{4}$ and ventricular CSF flow artifacts, ${ }^{12}$ were qualitatively described.
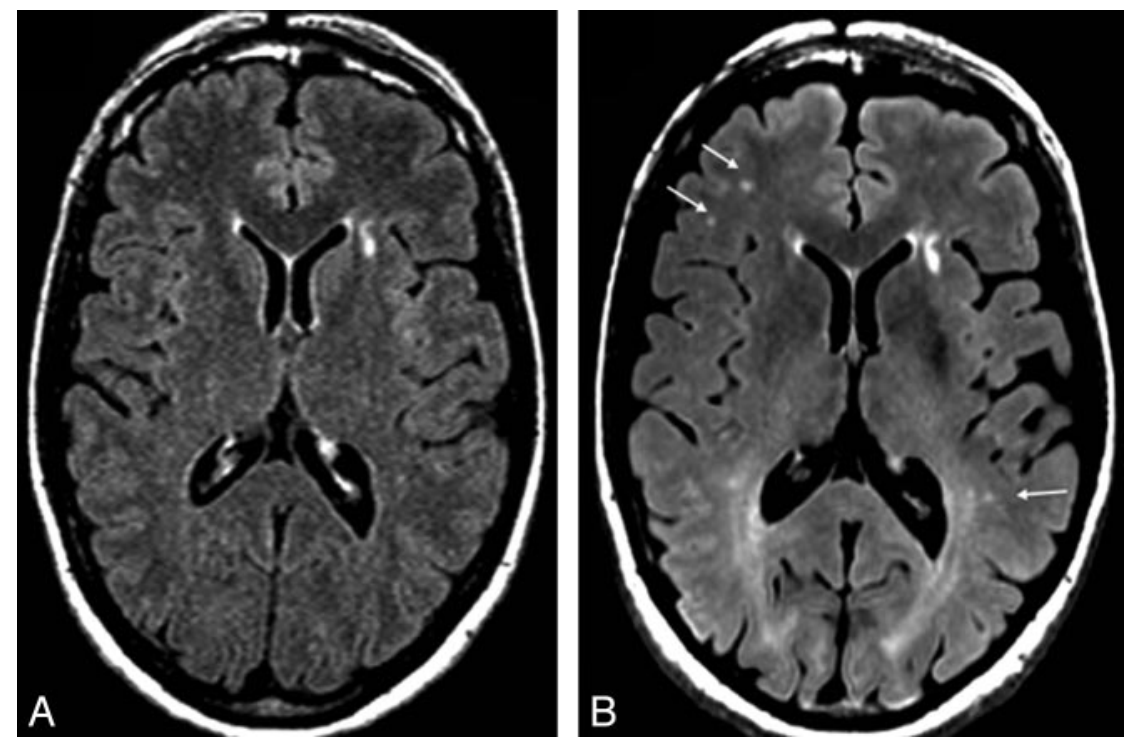

Fig 1. Comparison of $1.5 \mathrm{~T}$ and $3 \mathrm{~T}$ axial $2 \mathrm{D}$ FLAIR MR images in a 50-year-old healthy woman. Images of the same regions with equivalent pulse sequences display the improved sensitivity in discrete foci-detecting capabilities (arrows) and tissue resolution (tissue-CSF distinction) of the 3T scanner. Representative images are shown. In total, FLAIR showed 11 discrete foci in the whole brain (total brain volume of discrete hyperintense foci $=310 \mathrm{~mm}^{3}$ ) at $1.5 \mathrm{~T}$ and 26 discrete foci (total volume $=521 \mathrm{~mm}^{3}$ ) at $3 \mathrm{~T}$. The $1.5 \mathrm{~T}$ FLAIR scan $(A)$ shows less prominent number and conspicuousness of discrete foci than seen at $3 \mathrm{~T}(B)$. The arrows show some of the differences between the images. Note that anterior capping is present on both platforms However, a striking diffuse posterior white matter hyperintensity is present only at 3T, part of which is related to posterior capping. 


\begin{tabular}{|c|c|c|c|c|}
\hline & \multirow[b]{2}{*}{$1.5 \mathrm{~T}(n=15)$} & \multicolumn{3}{|c|}{ 3T vs $1.5 T^{*}(n=15)$, } \\
\hline & & $3 \mathrm{~T}(n=15)$ & $P$ Value & $3 \mathrm{~T}(n=22)$ \\
\hline No. discrete hyperintense foci & $5.46 \pm 9.15$ & $9.4 \pm 13.08$ & .0066 & $10.68 \pm 14.39$ \\
\hline Range & $0-33$ & $0-47$ & & $0-47$ \\
\hline No. subjects with $\geq 1$ discrete hyperintense focus & 10 & 10 & & 15 \\
\hline Total cerebral discrete hyperintense foci volume $\left(\mathrm{mm}^{3}\right)$ & $92.69 \pm 151.62$ & $170.33 \pm 242.51$ & .0054 & $188.43 \pm 257.58$ \\
\hline Range & $0-536.65$ & $0-871.68$ & & $0-871.68$ \\
\hline
\end{tabular}

* Wilcoxon signed rank test. See also Fig 2.

Table 3: Length/diameter of FLAIR discrete hyperintense foci

\begin{tabular}{lccc}
\hline & $1.5 \mathrm{~T}(n=15)$ & $3 \mathrm{~T}(\mathrm{n}=15)$ & $3 \mathrm{~T}(n=22)$ \\
\hline Maximum length/diameter $(\mathrm{mm})^{*}$ & $2.94 \pm 1.14$ & $2.75 \pm 1.22$ & $2.92 \pm 1.28$ \\
Range $(\mathrm{mm})$ & $1.24-8.36$ & $0.91-9.89$ & $0.75-9.89$ \\
\hline
\end{tabular}

${ }^{*}$ No significant group differences $(P=.68)$ when comparing 1.5T and $3 T$.

\section{Statistical Analysis}

The Spearman rank correlation analysis was used to assess the relationship between FLAIR discrete hyperintense foci number or volume and age. Wilcoxon signed rank test was used to assess the difference between age and discrete hyperintense foci detectability on the 1.5 and $3 \mathrm{~T}$ platforms. A modified Wilcoxon test ${ }^{13}$ for clustered data was used to assess the difference between discrete hyperintense foci length on the 1.5 and $3 \mathrm{~T}$ platforms. The Choi test ${ }^{14}$ assessed the differences between correlations on the $1.5 \mathrm{~T}$ versus $3 \mathrm{~T}$ platform. For all analysis, a $P$ value $<.05$ was considered statistically significant in this exploratory study.
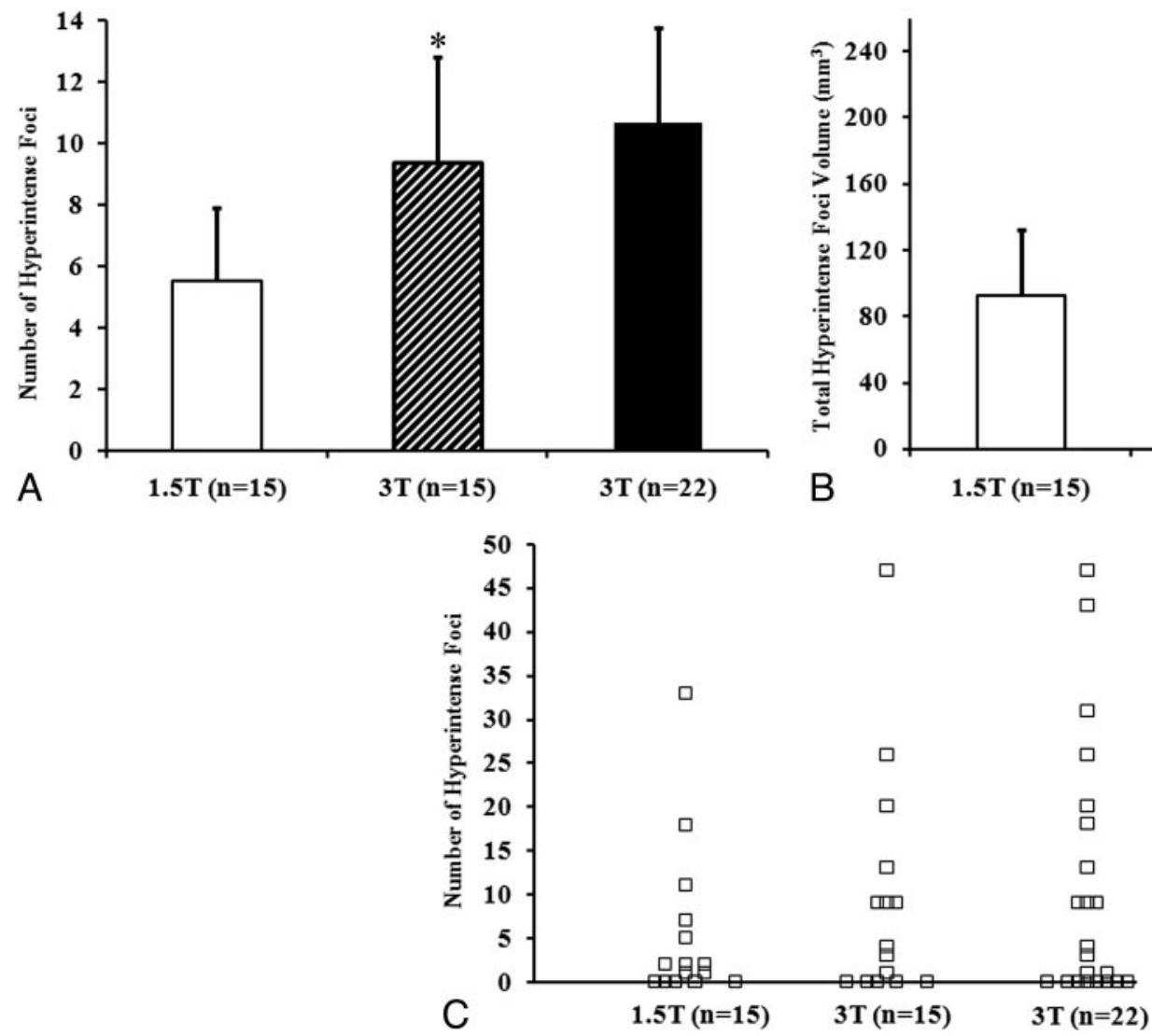

\section{Results}

\section{Discrete Parenchymal Hyperintense Foci}

In general, discrete hyperintense foci were small and punctate, linear, or curvilinear (Fig 1). In the 22 subjects scanned with 3T FLAIR, we found $10.68 \pm 14.39$ such foci (range, $0-47$ ), with each focus having a volume of $188.43 \pm 257.58 \mathrm{~mm}^{3}$ (range, $0-871.68 \mathrm{~mm}^{3}$ ) and a length of $2.92 \pm 1.28 \mathrm{~mm}$ (range, $0.759 .89 \mathrm{~mm}$ ) (Tables 2 and 3 ). Foci were present in 15 $(68 \%)$ of the 22 subjects at $3 \mathrm{~T}$ (Fig 2). Analysis revealed differences between the platforms; 3T FLAIR showed a larger num-

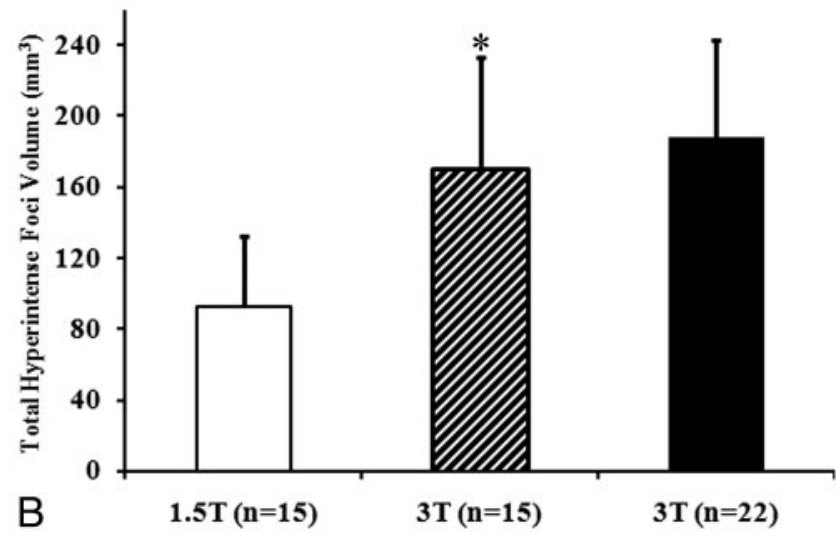

Fig 2. $A$ and $B$, Graphs show a larger hyperintense foci number $(A, P=.0066)$ and volume $(B, P=.0054)$ on $3 \mathrm{~T}$ FLAIR scans than on $1.5 \mathrm{~T}$ FLAIR scans. Histograms indicate mean; bars, standard error of the mean. Asterisks indicate a significant difference $(P<.05)$ when comparing $1.5 \mathrm{~T}$ with $3 \mathrm{~T}$ in the 15 subjects scanned on both platforms. $C$, Distribution of total brain hyperintense foci number for each subject; squares indicate individual cases. See also Table 2. 


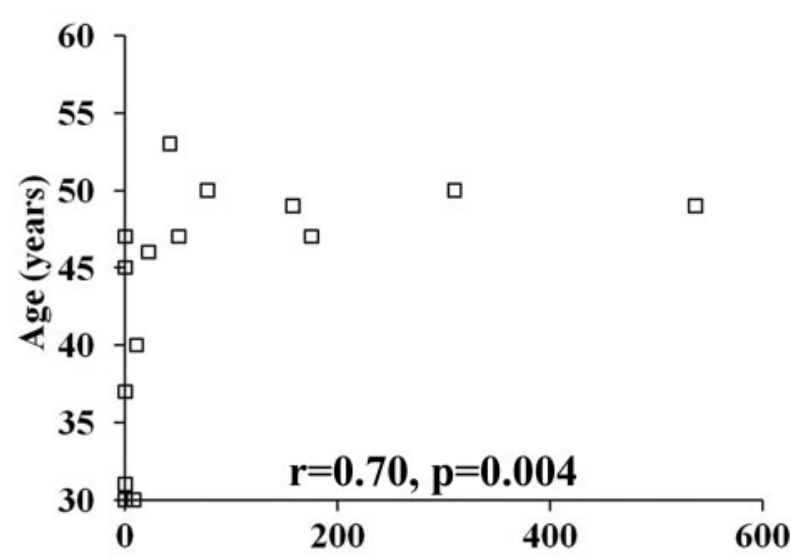

A

Total Hyperintense Foci Volume at $1.5 \mathrm{~T}\left(\mathrm{~mm}^{3}\right)$

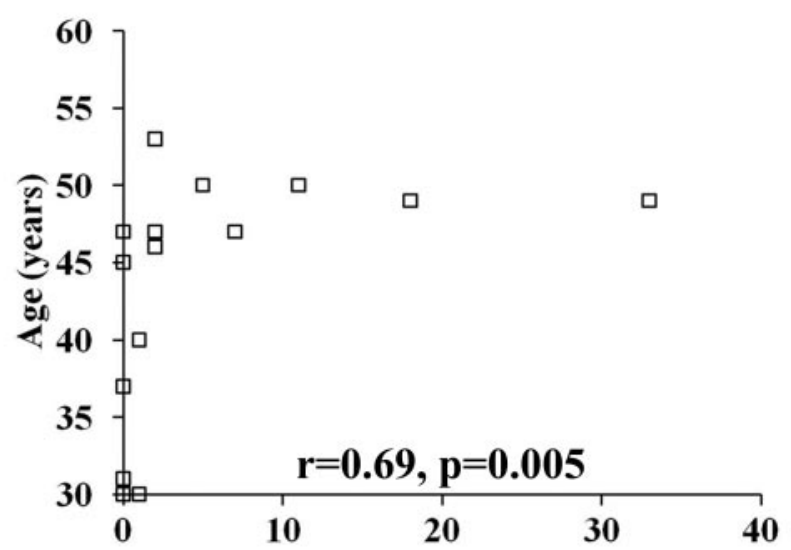

C

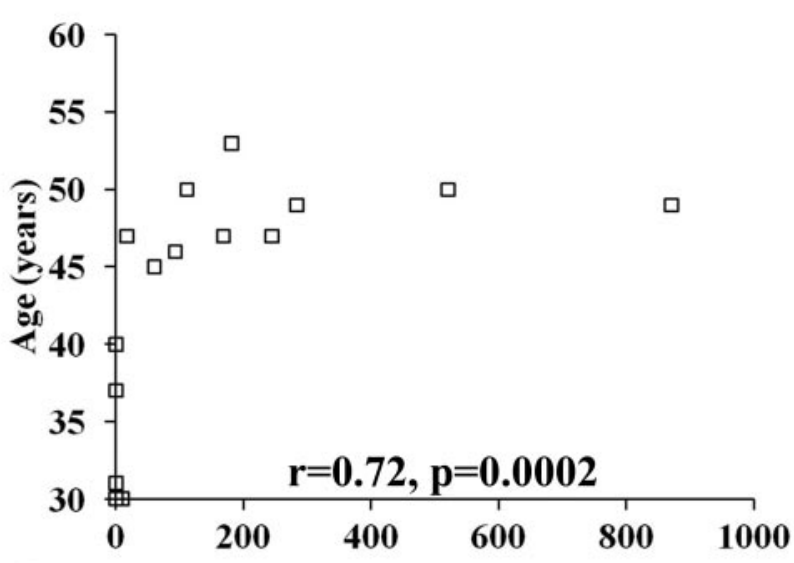

B Total Hyperintense Foci Volume at $3 \mathrm{~T}\left(\mathrm{~mm}^{3}\right)$

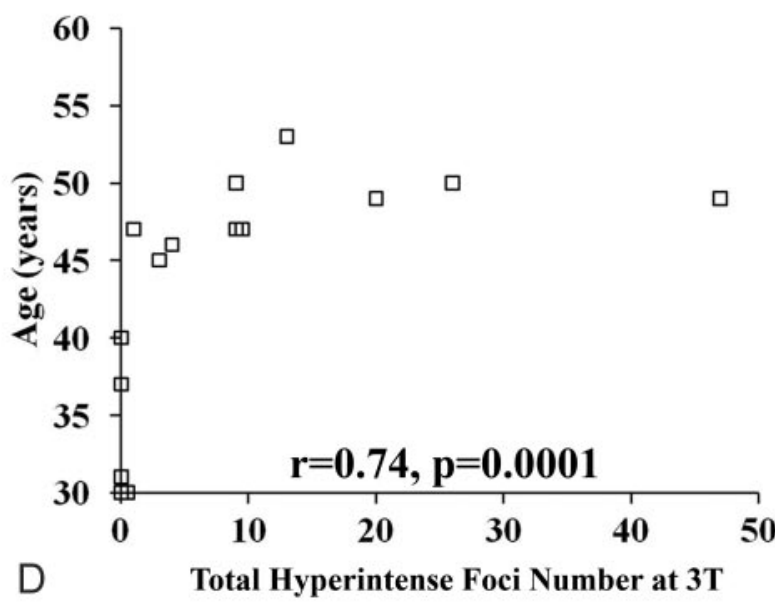

Fig 3. Relationship between total brain FLAIR hyperintense foci volume/number and age at 1.5 and $3 T$. Scatterplots show the relationship between total FLAIR hyperintense foci volume $(A$ and $B)$ or number $(C$ and $D)$ and age on 1.5 and $3 \mathrm{~T}$ scans in the healthy volunteers $(n=15)$. Each square represents a subject. Total FLAIR hyperintense foci volume $(r=0.70, P=$ $.004)$ and number $(r=0.69, P=.005)$ at $1.5 \mathrm{~T}$ and total FLAIR hyperintense foci volume $(r=0.74, P=.0001)$ and number $(r=0.72, P=.0002)$ at $3 \mathrm{~T}$ show a significant correlation with age. See also Table 4.

ber and increased global volume of discrete hyperintense foci compared with 1.5T FLAIR (Table 2 and Figs 1 and 2). However, there was no significant difference in the length/diameter of the individual discrete hyperintense foci at 1.5T (2.94 \pm $1.14 \mathrm{~mm}$; range, $1.24-8.36 \mathrm{~mm})$ versus $3 \mathrm{~T}(2.75 \pm 1.22 \mathrm{~mm}$; range, $0.91-9.89 \mathrm{~mm})(P=.68)$. Thus, the larger total hyperintense foci volume with 3 T FLAIR was driven by a larger number of small hyperintense foci, not an increased size of hyperintense foci already detected with $1.5 \mathrm{~T}$ FLAIR. Discrete hyperintense foci number $(1.5 \mathrm{~T}, r=0.69, P=.005$; 3T, $r=$ $0.74, P=.0001)$ and volume $(1.5 \mathrm{~T}, r=0.70, P=.004$; $3 \mathrm{~T}, r=$ $0.72, P=.0002$ ) correlated strongly with age (Table 4 and Fig $3)$. According to the Choi test for comparing correlations (Table 4), there was no difference in the correlation between age and number of hyperintense foci $(P=.2)$ or age and hyperintense foci volume $(P=.3)$ when comparing the 2 field strengths. No cortical/juxtacortical, callosal, or infratentorial discrete hyperintense foci were seen with either $1.5 \mathrm{~T}$ or $3 \mathrm{~T}$ FLAIR; all foci were localized in the deep or periventricular supratentorial white matter (Fig 1). Although some were close to the ventricles, none directly abutted the ventricular ependyma. The shape and morphology of the hyperintense foci were similar on both platforms.
Table 4: Brain FLAIR discrete hyperintense foci associated with age

\begin{tabular}{llc}
\hline & \multicolumn{2}{c}{ Correlation with Age } \\
\cline { 2 - 3 } & $r_{\mathrm{s}}$ & $P$ \\
\hline 1.5T discrete foci: total cerebral number & 0.69 & 0.005 \\
1.5T discrete foci: total cerebral volume & 0.70 & 0.006 \\
3T discrete foci: total cerebral number & 0.74 & 0.0001 \\
3T discrete foci: total cerebral volume & 0.72 & 0.0002 \\
\hline
\end{tabular}

Note:- $r_{\mathrm{s}}=$ Spearman correlation coefficient. According to Choi's test for correlations, there was no difference in the correlation between age and number of hyperintense foci $(p=0.2)$ or age and hyperintense foci volume $(p=0.3)$ when comparing the 2 scanners.
See also Fig 3 .

\section{Other Hyperintensities}

Capping hyperintensity immediately anterior to the frontal horns of the lateral ventricles was present on all scans (Figs 1 and 4). For the 22 subjects scanned with 3T FLAIR, the thickness of the capping appeared symmetric and thin $(1.48 \pm 0.42$ $\mathrm{mm}$; range, $0.90-2.54 \mathrm{~mm})$. The results were similar for the subgroup of 15 subjects scanned with both 3T FLAIR (1.42 \pm $0.35 \mathrm{~mm}$; range, $0.99-2.22 \mathrm{~mm}$ ) and 1.5T FLAIR (1.53 \pm $0.19 \mathrm{~mm}$; range, $1.23-2.05 \mathrm{~mm}$ ). Paired Wilcoxon analysis failed to detect a significant difference in anterior cap thickness between the field strengths $(P=.21)$.

Posterior periventricular capping hyperintensity was ob- 

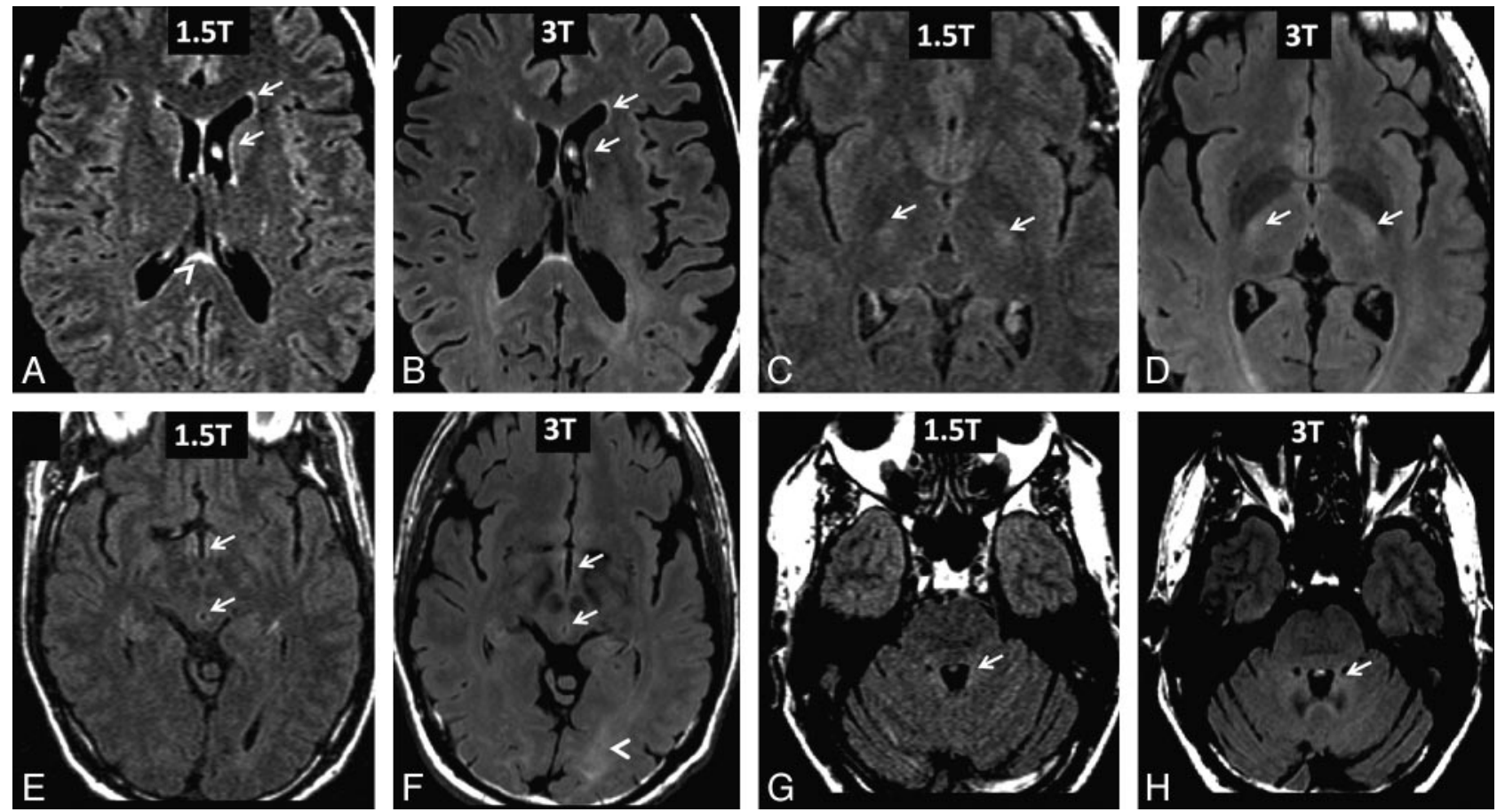

Fig 4. Comparison of $1.5 T$ and $3 T$ FLAIR images from healthy adult subjects. $A$ and $B$, Representative paired axial FLAIR images of a 47-year-old woman. Both platforms show a bright periventricular lining of the lateral ventricles (arrows) and septum pellucidum hyperintensity (arrowhead). Also note the prominent hyperintense CSF flow artifacts in the frontal horn of the left lateral ventricle (arrows). $C$ and $D$, Representative paired axial FLAIR images of a 50-year-old woman. Both platforms show bilateral corticospinal tract hyperintensity (arrows). $E$ - $H$, Representative paired axial FLAIR images of a 53-year-old man. Both platforms show hyperintensities (arrows) of the third ventricular lining $(E$ and $F$, cerebral aqueduct lining ( $E$ and $F$, and within the fourth ventricle due to CSF flow ( $G$ and $H$ ). Ventricular CSF pulsation artifacts are seen to a similar degree between platforms, but only $3 T$ FLAIR shows diffuse posterior parenchymal white matter hyperintensity $(F$, arrowhead).

served in all subjects with 3 T FLAIR (Figs $1 B$ and $4 B, D-F$ ). This referred to both a thin line and hazy area of hyperintensity adjacent to the occipital horns of the lateral ventricles. Although faint posterior capping was also observed with $1.5 \mathrm{~T}$ FLAIR (Figs $1 A$ and $4 A$ ), it was more prominent with $3 \mathrm{~T}$ FLAIR. In addition, a diffuse posterior parenchymal white matter hyperintensity was present on 3T FLAIR scans of all subjects (Figs $1 B$ and $4 B, D-F$ ). This diffuse posterior white matter hyperintensity was not seen in any subject with $1.5 \mathrm{~T}$ FLAIR (Figs $1 A$ and $4 A, C, E$ ).

Flow artifacts, such as CSF pulsation, were similar in both platforms and included hyperintensities in the third and fourth ventricles (Figs 1 and 4). Bilateral and symmetric hyperintensity of the corticospinal tract (Fig $4 A-F)$ and a thin periventricular lining around the lateral ventricles (Figs 1 and $4 A-H$ ), third ventricle (Fig $4 E, F$ ) and fourth ventricle (Fig $4 G$, $H$ ), and cerebral aqueduct (Fig $4 E, F$ ) were present in all cases and were seen to a similar extent and frequency with $1.5 \mathrm{~T}$ and 3T FLAIR MR imaging. Septum pellucidum hyperintensity was also observed to a similar extent and frequency at both platforms (Fig 4A, B).

\section{Discussion}

The present study demonstrates that healthy controls commonly display a wide variety of hyperintensities on 3T FLAIR images, which include all of those previously known at $1.5 \mathrm{~T}$. However, the 3T platform shows several of the hyperintensities as more numerous and prominent, such as discrete hyperintense foci and diffuse posterior white matter hyperintensity. These normative findings should be taken into account when interpreting 3T FLAIR images of patients with suspected pathologic conditions.

It was striking how common and numerous discrete hyperintense foci were on the 3T FLAIR scans. Most (68\%) of the subjects' 3T FLAIR scans revealed discrete hyperintense foci, with an average of 10.68 of these foci per subject. The total cerebral number and volume of discrete hyperintense foci were significantly higher on 3T FLAIR versus $1.5 \mathrm{~T}$ FLAIR scans, both nearly double at 3T. However, the discrete hyperintense foci were confined to previously known locations ${ }^{4}$ for incidental hyperintensities in healthy individuals (ie, the deep periventricular white matter with sparing of the corpus callosum, cortical, juxtacortical, and posterior fossa regions). In addition, the size and morphology were similar to what has been described previously ${ }^{4}$ at lower field strengths-small and punctate, linear, or curvilinear. The underlying pathology relating to these hyperintense foci is unknown but most likely represents a heterogeneous collection of age-related changes. ${ }^{15-17}$ One finding was of particular interest because it was seen only on 3T FLAIR scans-diffuse posterior white matter hyperintensity. This was seen in all 22 cases and may represent normal hypomyelination relative to the anterior white matter, leading to higher water content. ${ }^{18,19}$ This would be consistent with the known development of cerebral white matter in which the posterior area is the latest to myelinate and usually does so incompletely. ${ }^{19,20}$ Alternative explanations include pathologic changes with aging such as demyelination and gliosis.

Several other findings including periventricular capping, septal hyperintensity, CSF flow artifacts, and hyperintensity of 
the corticospinal tracts were seen on 3T FLAIR scans to a similar character and extent than present at 1.5T. The above-mentioned findings were consistent with previous reports of incidental hyperintensities on FLAIR images. ${ }^{4,12,21,22}$

There are several limitations in our study. Because of the small sample size, the findings should be considered preliminary. Because subject enrollment was based solely on interview, we cannot exclude the possibility that some of the patients had undetected illnesses in a presymptomatic stage (though most volunteers were receiving regular health maintenance with a primary care physician). We did not compare the findings in our healthy volunteers with those of patients with known neurologic diseases such as MS and cerebrovascular disease. Such comparisons will be vital to understanding how the $3 \mathrm{~T}$ platform affects the specificity of findings. There were small differences in section thickness in the MR imaging protocol among the subjects scanned at $3 \mathrm{~T}$. However, this did not seem to influence the results and conclusions of the study. Finally, the $1.5 \mathrm{~T}$ versus 3T FLAIR comparison was hampered by differences in vendors and other scan parameters, though we attempted to make the protocols as similar as possible, given the hardware and software differences.

Our findings have several implications for patient care. The simple presence of hyperintense foci, even in large numbers, or the presence of posterior white matter hyperintensity should not necessarily be interpreted as reflecting a pathologic condition, because these may occur in the healthy population. Although 3T FLAIR has the potential to become a more accurate investigative tool than 1.5T FLAIR, new standards will have to be developed and caution should be exercised when applying 3T FLAIR MR imaging to the study of neurologic disorders such as MS when determining the clinical relevance of detected hyperintensities. It is likely that the size, morphology, and location of hyperintensities will help to guide clinical decisions. Additional studies should be undertaken, with a larger number of subjects and comparisons with abnormal conditions, to extend and confirm our findings.

\section{Conclusions}

Brain 2D FLAIR MR imaging at 3T commonly shows a range of discrete and diffuse hyperintensities in healthy individuals that are more prominent than those with FLAIR at 1.5T. When interpreting 3T FLAIR scans of the brain in patients with suspected cerebral disorders, one should be mindful of hyperintensities that may represent "normal" rather than pathologic findings.

\section{References}

1. Neema M, Stankiewicz J, Arora A, et al. MRI in multiple sclerosis: what's inside the toolbox? Neurotherapeutics 2007;4:602-17

2. Bakshi R, Thompson AJ, Rocca MA, et al. MRI in multiple sclerosis: current status and future prospects. Lancet Neurol 2008;7:615-25

3. Stevenson VL, Gawne-Cain ML, Barker GJ, et al. Imaging of the spinal cord and brain in multiple sclerosis: a comparative study between fast FLAIR and fast spin echo. J Neurol 1997;244:119-24

4. Gawne-Cain ML, Silver NC, Moseley IF, et al. Fast FLAIR of the brain: the range of appearances in normal subjects and its application to quantification of white-matter disease. Neuroradiology 1997;39:243-49

5. Phal PM, Usmanov A, Nesbit GM, et al. Qualitative comparison of 3-T and 1.5-T MRI in the evaluation of epilepsy. AJR Am J Roentgenol 2008;191:890-95

6. Alvarez-Linera J. 3T MRI: advances in brain imaging. Eur J Radiol 2008;67:415-26

7. Keiper MD, Grossman RI, Hirsch JA, et al. MR identification of white matter abnormalities in multiple sclerosis: a comparison between $1.5 \mathrm{~T}$ and $4 \mathrm{~T}$. AJNR Am J Neuroradiol 1998;19:1489-93

8. Sicotte NL, Voskuhl RR, Bouvier S, et al. Comparison of multiple sclerosis lesions at 1.5 and 3.0 Tesla. Invest Radiol 2003;38:423-27

9. Wattjes MP, Harzheim M, Kuhl CK, et al. Does high-field MR imaging have an influence on the classification of patients with clinically isolated syndromes according to current diagnostic MR imaging criteria for multiple sclerosis? AJNR Am J Neuroradiol 2006;27:1794-98

10. Papadakis NG, Martin KM, Mustafa MH, et al. Study of the effect of CSF suppression on white matter diffusion anisotropy mapping of healthy human brain. Magn Reson Med 2002;48:394-98

11. Boss A, Graf $\mathrm{H}$, Berger A, et al. Tissue warming and regulatory responses induced by radio frequency energy deposition on a whole-body 3-Tesla magnetic resonance imager. J Magn Reson Imaging 2007;26:1334-49

12. Bakshi R, Caruthers SD, Janardhan V, et al. Intraventricular CSF pulsation artifact on fast fluid-attenuated inversion-recovery MR images: analysis of 100 consecutive normal studies. AJNR Am J Neuroradiol 2000;21:503-08

13. Datta S, Satten GA. Rank-sum tests for clustered data. J Am Stat Assoc 2005;100:908-15

14. Choi SC. Tests of equality of dependent correlation coefficients. Biometrika 1997;64:645-47

15. Kirkpatrick JB, Hayman LA. White-matter lesions in MR imaging of clinically healthy brains of elderly subjects: possible pathologic basis. Radiology 1987;162:509-11

16. Liao D, Cooper L, Cai J, et al. The prevalence and severity of white matter lesions, their relationship with age, ethnicity, gender, and cardiovascular disease risk factors: the ARIC Study. Neuroepidemiology 1997;16:149-62

17. Young VG, Halliday GM, Kril JJ. Neuropathologic correlates of white matter hyperintensities. Neurology 2008;71:804-11. Epub 2008 Aug 6

18. Murakami JW, Weinberger E, Shaw DW. Normal myelination of the pediatric brain imaged with fluid-attenuated inversion-recovery (FLAIR) MR imaging. AJNR Am J Neuroradiol 1999;20:1406-11

19. Ashikaga R, Araki Y, Ono Y, et al. Appearance of normal brain maturation on fluid-attenuated inversion-recovery (FLAIR) MR images. AJNR Am J Neuroradiol 1999;20:427-31

20. Holland BA, Haas DK, Norman D, et al. MRI of normal brain maturation. AJNR Am J Neuroradiol 1986;7:201-08

21. Sze G, De Armond SJ, Brant-Zawadzki M, et al. Foci of MRI signal (pseudo lesions) anterior to the frontal horns: histologic correlations of a normal finding. AJR Am J Roentgenol 1986;147:331-37

22. Naganawa S, Koshikawa T, Nakamura T, et al. Comparison of flow artifacts between 2D-FLAIR and 3D-FLAIR sequences at 3 T. Eur Radiol 2004;14:190108. Epub 2004 Jun 19 